\title{
Isolation \& Identification of FAV-4 Tianjin Strain and Pathogenicity to Chicks
}

\author{
Rui Liu, Jing Wang, Fugui Li* \\ College of Animal Science and Veterinary Medicine, Tianjin Agricultural University, Tianjin, China \\ Email address: \\ m13331710020@163.com (Rui Liu),wangjingtn2014@126.com (Jing Wang), lifugui2008163@163.com(Fugui Li) \\ ${ }^{*}$ Corresponding author
}

To cite this article:

Rui Liu, Jing Wang, Fugui Li. Isolation \& Identification of FAV-4 Tianjin Strain and Pathogenicity to Chicks. Animal and Veterinary Sciences. Vol. 7, No. 1, 2019, pp. 12-17. doi: 10.11648/j.avs.20190701.12

Received: January 10, 2019; Accepted: February 27, 2019; Published: March 20, 2019

\begin{abstract}
The Hydropericardium-hepatitis Syndrome (HHS) caused by Fowl adenovirus 4 (FAV-4) is a new epidemic disease in recent years and firstly reported in Tianjin, 2016. The isolation, identification and pathogenicity of FAV-4 Tianjin strain were studied in the paper for providing the theoretical basis to the law of occurrence, development and termination. The virus was isolated and identified from dead \& sick birds on a chicken farm in Tianjin by chicken embryo inoculation with tissue homogenate supernatant, PCR and sequencing experiment with viral allantoic fluid; And the pathogenicity was proceeded by inoculating the isolate into 100 one-day-old chicks, which divided into group A, B, C (experimental group) and group D (control), the symptoms, mortality and morphological changes were observed daily, meanwhile the virus was detected by PCR to be sure the same as inoculating strain. The results show that the isolate was $665 \mathrm{bp}$ in electrophoresis, had homology of $98.3 \%-97.3 \%$ with FAV-4 by sequencing analysis and finally named as FAV-4 TJ1607 strain in GenBank; They also show in Pathogenicity research that the symptoms were appeared on the 3 nd day, death on the 4 th day and the peak of death on the 5 th day and end of the 8th day in group A and B; The mortality was $64 \%$ in group A and $72 \%$ in group B; Hepatic edema \& congestion, large amounts of basophilic intranuclear inclusions, hydropericardium, parechymal myocarditis and pulmonary edema were observed obviously on the 4th day in group A and B; ALL above changes were the same in group C just appeared 3 days later except death trend and mortality for 32\%; And FAV-4 TJ1607 was detected in all experimental groups. The measures of prevention and treatment will be recommended in poultry industry accordance with the HHS disease model.
\end{abstract}

Keywords: Isolation, Identification, FAV-4 TJ1607 Strain, Pathogenicity, HHS Disease Model

\section{Introduction}

The Hydropericardium-hepatitis Syndrome (HHS) caused by Fowl adenovirus 4 (FAV-4) which belongs to the adenoviral family, avian adenovirus is a new epidemic disease in recent years [1]. FAV-4 is widely present in respiratory and digestive tracts of various poultry in the world, chickens were insidiously infected or the virus brought on morbidity and mortality act as secondary pathogen [2]. HHS characterized by sudden onset, rapid death, hydropericardium obviously and hepatitis with large amounts of basophilic intranuclear inclusions was firstly reported in Tianjin in July 2016, and then became an important infectious disease in poultry industry [3]. The molecular biology of FAV-4 had been studied in past two years but rarely known about its pathogenicity.
Animal disease models are used to three researches, nowadays, including experimental pathology, experimental physiology and experimental therapeutics [4]. The pathogenicity of FAV-4 Tianjin strain followed by isolation and identification was studied in the paper for understanding the law of occurrence, development and termination or pathogenic mechanism for providing the theoretical basis on subsequent prevention and treatment of HHS.

\section{Materials and Methods}

\subsection{Virus}

FAV-4 Tianjin strain (TJ1607) was named in our Lab for research of pathogenicity, followed isolation and identification of pathogen including epidemiological 
investigation, necropsy, morphological observation and virological examination from a chicken farm; Chicken embryo allantoic fluid was collected by chicken embryo experiment with tissue homogenate supernatant from natural case; Liver homogenate supernatant was acquired by natural case.

\subsection{Experimental Animals}

15 dead \& sick chickens from poultry farm in Tianjin on July 18, 2016; 20 Ten-day-old SPF chicken embryos; 100 One-day-old healthy chicks (broiler).

\subsection{Experiment for Pathogenic Isolation}

The liver and spleen were sampled aseptically from 15 dead $\&$ sick chickens, homogenized with $1 \%$ ceftiofur sodium and centrifuged for superabundant. The supernatant was inoculated into 20 ten-day-old SPF chicken embryos with $0.1 \mathrm{~mL} / \mathrm{embryo}$, incubated at $37^{\circ} \mathrm{C}$ and the allantoic fluid was collected for virus detection at $48 \mathrm{~h}$ post-inoculation. Agarose gel electrophoresis was proceeded with tissue homogenate supernatant and allantoic fluid. The result was observed under UV light and photographed.

\subsection{Experiment for Pathogenic Identification}

The viral DNA was extracted from the tissue homogenate supernatant and allantoic fluid by the method of genomic DNA extraction kit (DP315), amplified by PCR according to the specific primers and purified in our laboratory; And then the purified product was submitted to Shanghai Biotech Biotechnology Service Company for sequencing. The isolate was analyzed on homology and genetic evolution by Megalign of DNA star software and 11 domestic and foreign reference strains in GenBank.

\subsection{Experiment for Pathogenicity}

\subsubsection{Experiment for Challenge}

One hundred 1-day-old chicks were randomly divided into 4 groups named group A, B, C, and group D. Group A and group B were challenged and inoculated with viral allantoic fluid and liver homogenate supernatant by dropping nose, eye and orally, which saline was administrated into chicks in group $\mathrm{C}$ and $\mathrm{D}$. The chicks in group $\mathrm{C}$ were mixed rearing in group A and B 3 days post-inoculation. The details will be seen in table 1.

Table 1. Experimental design.

\begin{tabular}{|c|c|c|c|c|}
\hline Groups & Experiment & Dosage & Number of chicks & Notes \\
\hline Group A & Viral allantoic fluid & $0.1 \mathrm{~mL}$ & 25 & \\
\hline Group B & Liver homogenate supernatant & $0.1 \mathrm{~mL}$ & 25 & \\
\hline Group C & Normal saline & $0.1 \mathrm{~mL}$ & 25 & Mixed rearing in group A and B 3 days post-inoculation \\
\hline
\end{tabular}

\subsubsection{Experiment for Morbidity}

All birds were raised ordinarily, clinical appearances were observed daily and recorded carefully for incidence statistics, including mental conditions, drinking, feeding, feces and feather condition.

\subsubsection{Experiment for Mortality}

All dead birds were record in time, number, groups for fatality statistics, then autopsy for morphological and virological examination.

\subsubsection{Experiment for Macro-morphological Examination}

One chick from each group daily and all dead birds were autopsied according to poultry necropsy operation for general morphological observation. All tissue and organs were observed carefully especially heart, liver, spleen, lung et.al [5-6]. Simultaneously, some tissues were sampled for histopathological examination and virological tests.

\subsubsection{Experiment for Histopathological Examination}

Parts of heart, liver and lung were taken and fixed in $10 \%$ neutral formalin solution. The tissues were trimmed, $24 \mathrm{hrs}$ later, dehydrated, transparented, wax immersed, embedded, sliced off $3 \mu \mathrm{m}$, stained with hematoxylin-eosin and mounted. Morphological changes were observed under microscope.

\subsubsection{Experiment for Antigenic Mapping of TJ1607}

Liver and pericardial effusion were freezed in $-80^{\circ} \mathrm{C}$ refrigerator and then extracted total viral DNA, amplified by polymerase chain reaction and agarose gel electrophoresis. The result was observed under UV light and photographed.

\section{Results}

\subsection{Results of Pathogenic Isolation}

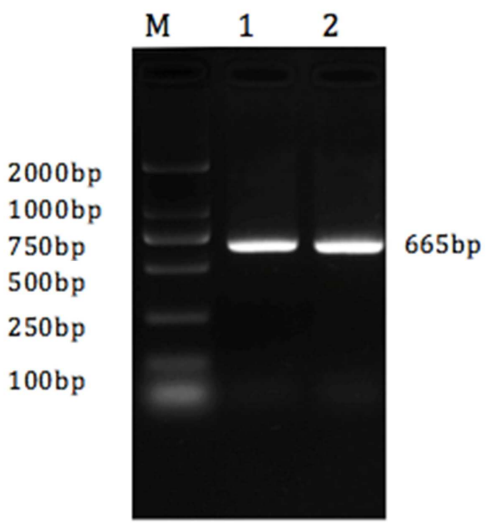

M: DL2501 DNA Maker 1: tissue homogenate supernatant 2: allantoic fluid

Figure 1. Results of electrophoresis with isolate.

The results of electrophoresis derived from two samples 
were all 665 bp target band in DNA maker, which No 1 was tissue homogenate supernatant and No 2 was amplified allantoic fluid (Figure 1). The isolate belongs to fowl adenovirus.

\subsection{Results of Pathogenic Identification}

The isolate had homology of $98.3 \%-97.3 \%$ in homology analysis by Megalign compared with FAV-4 published in GenBank, 98.3\% with Chinese strain, 97.9\% with Indian strain, $97.3 \%$ with Canadian strain, 93.3\% with adenovirus type 10 strain and 48.4\%-73.2\% homology with other adenovirus serotypes. The isolate was FAV-4 Tianjin strain which finally named as TJ1607 in GenBank (genebank accession number: KY885021). The results shown in figure 2.

Percent Identity

\begin{tabular}{|c|c|c|c|c|c|c|c|c|c|c|c|c|c|}
\hline & 1 & 2 & 3 & 4 & 5 & 6 & 7 & 8 & 9 & 10 & 11 & & \multirow{12}{*}{$\begin{array}{l}\text { EU177545.1 } \\
\text { Y09598.1 } \\
\text { U46933.1 } \\
\text { AF074946.1 } \\
\text { KM096544.1 } \\
\text { GU188428.1 } \\
\text { U26221.1 } \\
\text { KC493646.1 } \\
\text { GU734104.1 } \\
\text { AF083975.2 } \\
\text { TJ16007 }\end{array}$} \\
\hline 1 & & 50.8 & 75.4 & 48.8 & 99.8 & 98.7 & 95.8 & 72.5 & 74.1 & 69.1 & 97.9 & 1 & \\
\hline 2 & 82.0 & & 38.8 & 51.6 & 38.2 & 37.7 & 49.5 & 39.0 & 39.0 & 39.6 & 53.9 & 2 & \\
\hline 3 & 29.9 & 130.7 & & 40.3 & 53.2 & 52.4 & 75.3 & 54.8 & 56.6 & 55.3 & \begin{tabular}{|l|}
73.1 \\
\end{tabular} & 3 & \\
\hline 4 & 90.4 & 79.3 & 123.8 & & 39.2 & 39.2 & 47.2 & 40.4 & 39.7 & 41.0 & 48.1 & 4 & \\
\hline 5 & 0.2 & 134.2 & 73.9 & 130.4 & & 98.3 & 95.6 & 54.6 & 55.6 & 54.2 & 98.3 & 5 & \\
\hline 6 & 1.3 & 136.9 & 76.0 & 130.4 & 1.7 & & 95.6 & 53.8 & 55.0 & 53.5 & 97.3 & 6 & \\
\hline 7 & 4.3 & 85.5 & 30.1 & 95.8 & 4.5 & 4.5 & & 70.4 & 72.8 & 68.4 & 93.2 & 7 & \\
\hline 8 & 34.5 & 129.8 & 69.7 & 124.3 & 70.2 & 72.3 & 37.8 & & 63.9 & 62.8 & 69.6 & 8 & \\
\hline 9 & 31.8 & 129.8 & 65.3 & 129.1 & 67.7 & 69.3 & 33.8 & 49.5 & & 71.7 & 72.0 & 9 & \\
\hline 10 & 40.3 & 126.2 & 68.8 & 120.6 & 71.3 & 73.3 & 41.4 & 51.8 & 35.8 & & 65.1 & 10 & \\
\hline 11 & 2.1 & 7300 & 33.6 & 97.3 & 1.8 & 2.7 & 7.2 & 39.2 & 35.3 & 47.2 & & 11 & \\
\hline & 1 & 2 & 3 & 4 & 5 & 6 & 7 & 8 & 9 & 10 & 11 & & \\
\hline
\end{tabular}

Figure 2. Comparison of nucleotide sequence homology with FAV hexon gene.

Genetic evolution analysis of TJ1607 strain was proceeded by Megalign since it was closely related to FAV-4. The results shown in figure 3.

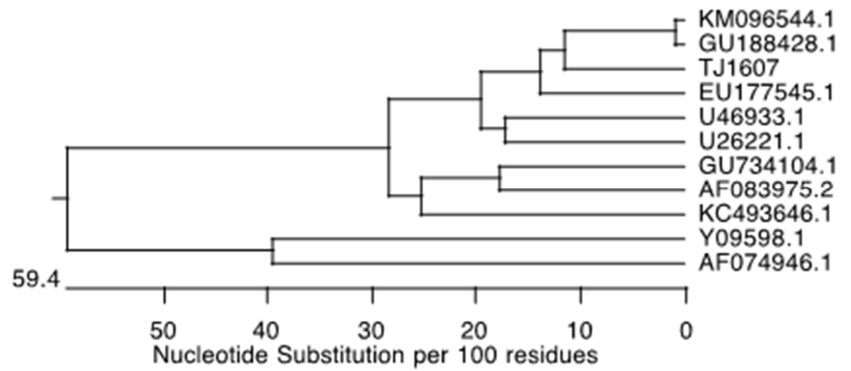

Figure 3. Genetic evolution analysis on hexon gene of adenovirus.

\subsection{Results of Pathogenicity}

\subsubsection{Results of Morbidity}

Clinical signs were in consistency in group A and B mainly from 3rd day post-inoculation whereas similar symptoms in group $\mathrm{C}$ just 3 days later of mixture in experimental groups. Symptoms were manifested as depression, loss of appetite, lethargy, yellow loose feces and others (Figure 4). There were no any symptoms in group D. All above symptoms are consistent with them in natural cases.

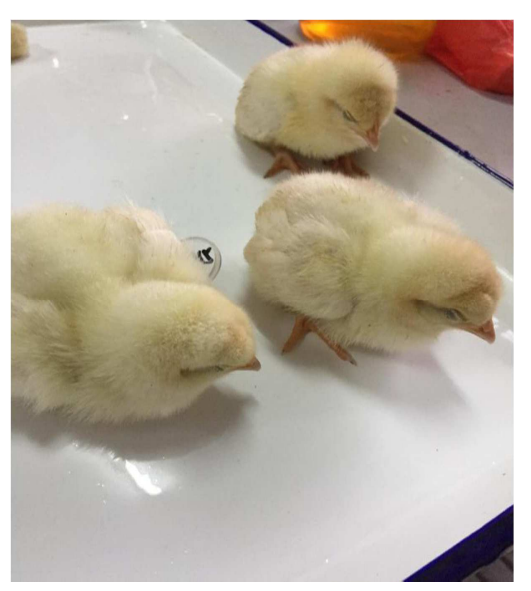

Figure 4. Mental status of affected chickens.

\subsubsection{Results of Mortality}

The first dead bird was occurred on the 4th day, peak reached on the 5th day and ended on the 8th day in group A and B. Death in group $\mathrm{C}$ started from the 3rd day post-mixed, peaked on the 4th day and ended on the 5th day. There was no dead bird in group D (control). The situation of occurrence and mortality shown in table 2 and the law of death shown in figure 5 .

Table 2. Situation of occurrence and mortality.

\begin{tabular}{lllll}
\hline Groups & Time of onset & Time of first death & Time of peak & Time of end \\
\hline Group A & 3rd day & 4th day & 5th day & 8 th day \\
Group B & 3rd day & 4th day & 5 th day & $84 \%$ \\
Group C (post-mixed ) & 3rd day & 3rd day & 4th day & $72 \%$ \\
Group D (control) & No & No & No & 5 th day \\
\hline
\end{tabular}




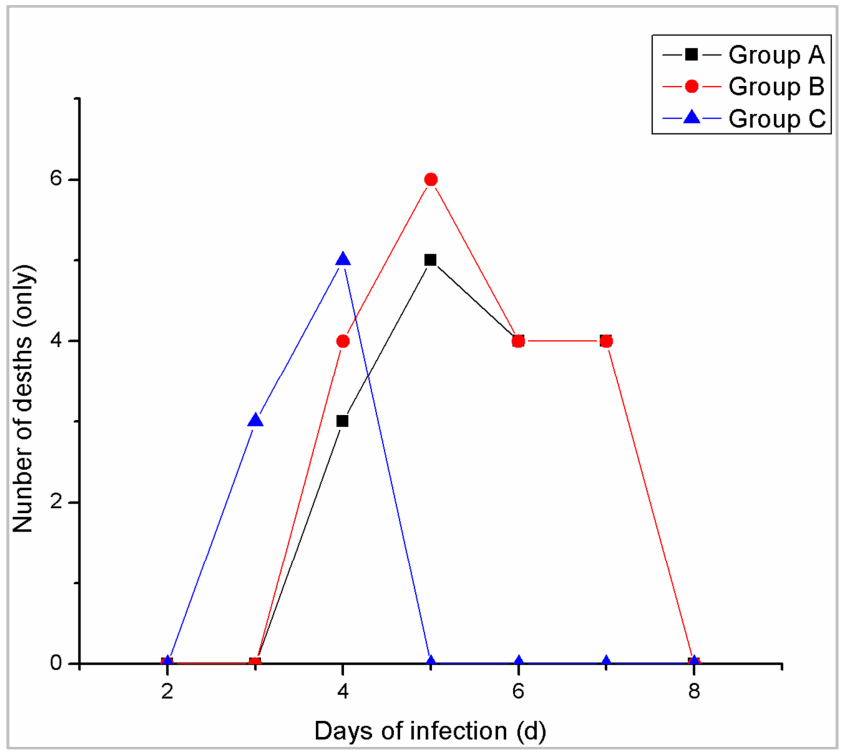

Figure 5. Pattern of death.

\subsubsection{Results of Macro-morphological Examination}

Gross lesions were observed in macro-morphological examination. Liver was swollen, moisture \& shimmery, yellowish, crisp accompanied by varying degrees of congestion and bleeding (Figure 6). The heart was yellowish and there was a large amount of light-yellowish transparent liquid in pericardium, hydropericardium, seen in Figure 7. Pulmonary edema and congestion were very obviously. There were no specific visible findings in other tissues and organs. The above gross lesions were consistent with them in natural cases.

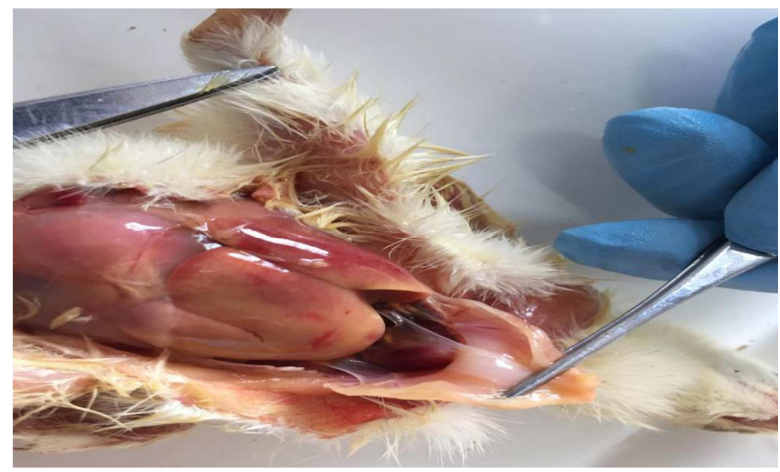

Figure 6. Gross lesions in chicken liver.

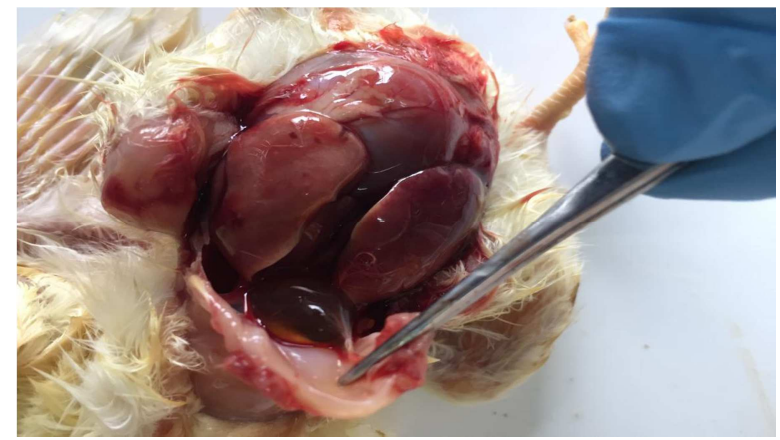

Figure 7. Hydropericardium in affected chicks.

\subsubsection{Results of Histopathological Examination}

Hepatic edema, congestion, disordered arrangement of hepatic cords, infiltration of inflammatory cells, degeneration and necrosis of hepatocytes with massive basophilic intranuclear inclusion bodies were observed in livers under microscopy (Figure 8, 9). Obvious interstitial edema, myocardial fiber breaks, necrosis, bleeding and plenty of inflammatocyte infiltration were seen in heart which was typical viral parechymal myocarditis (Figure 10). Plenty of exudates, severe congestion and few of inflammatory cell in lungs were prominent histopathological changes (Figure 11). The above histopathological changes which consistent with clinical cases were observed obviously on the 4th day in group $\mathrm{A}$ and $\mathrm{B}$ and the same in group $\mathrm{C}$ just appeared 3 days later.

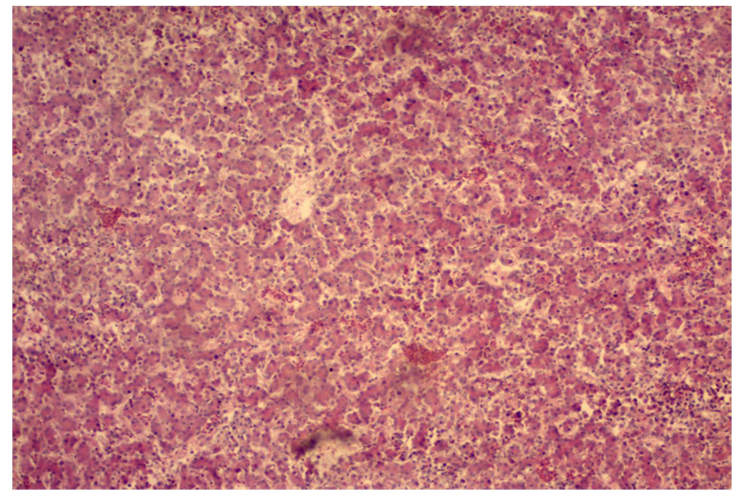

Figure 8. $(H E \times 100)$ Edema, congestion and inflammatocyte infiltration.

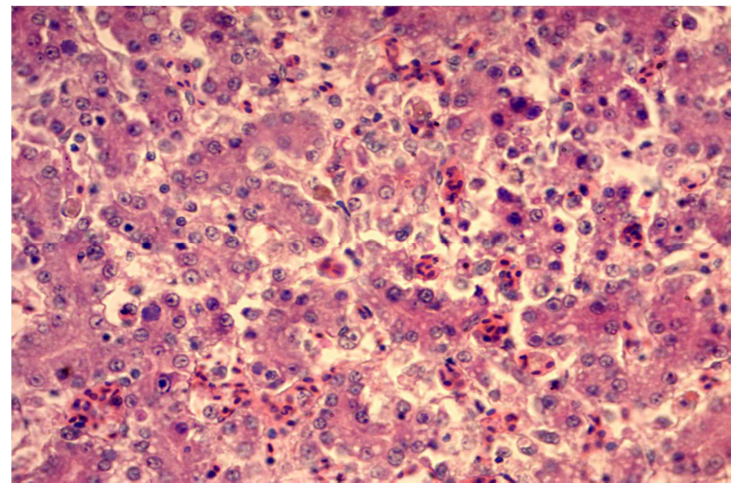

Figure 9. $(H E \times 400)$ Hepatocytic degeneration and intranuclear inclusion bodies.

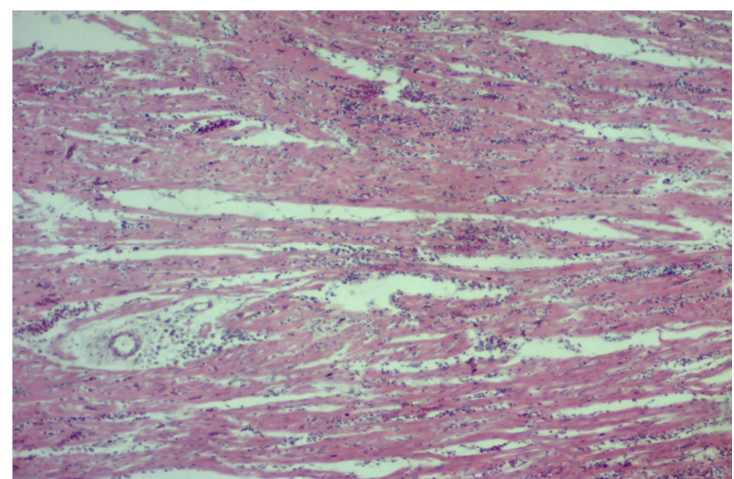

Figure 10. $(H E \times 100)$ Severe viral parechymal myocarditis. 


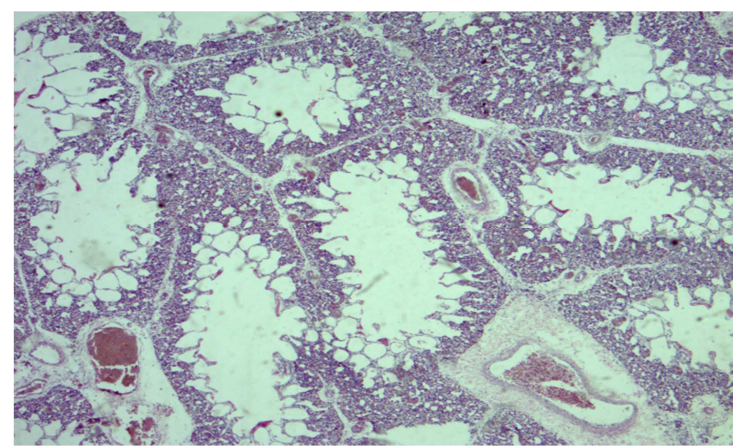

Figure 11. $(H E \times 100)$ Pulmonary edeme and congestion in lung.

\subsubsection{Results of Antigenic Mapping of TJ1607}

The electrophoresis results of four extractions from liver and pericardial effusion were all $665 \mathrm{bp}$ target band, which consistent with them in natural disease. It indicated that TJ1607 was detected in challenged chicks (Figure 12). It also evidenced that the results of pethogenicity could be used to clinical practice for disease control in poultry industry.

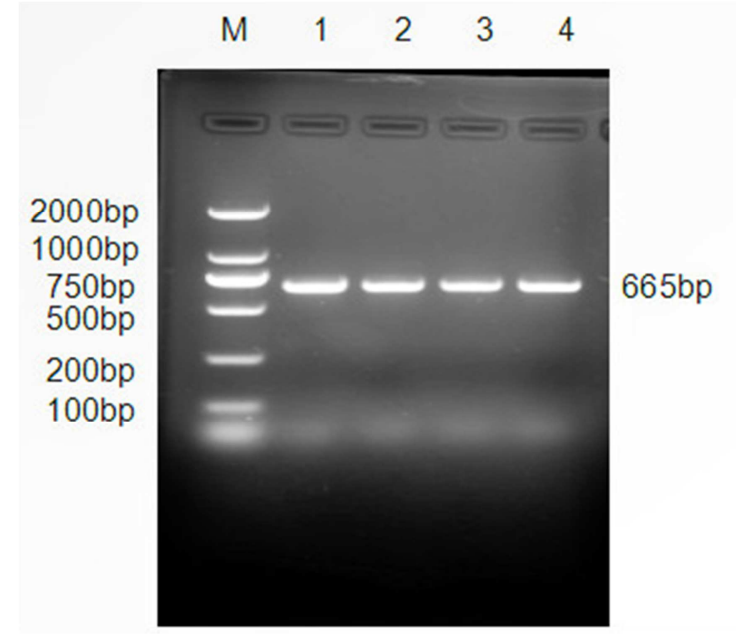

M:DL2501 DNA Maker 1.2: Liver tissue 3.4: Hydropericardium

Figure 12. Results of electrophoresis with FAV-4 TJ1607.

\section{Discussion}

\subsection{Pathogen Analysis}

HHS characterized by hydropericardium and inclusion body hepatitis is a highly pathogenic infectious disease firstly reported in Pakistan's Ankara region, and then in China in 2013 [7]. The disease has been widespread, since June 2015, in Hunan, Jiangsu, Shandong, Hebei, Liaoning and Jilin provinces in China and developed a type of chicken pericardial effusion and inclusion bodies hepatitis. HHS shows acute onset, spreads rapidly, mainly occur in 3 to 6 weeks-old chickens, especially broilers. At present, laying hens, breeders, ducks, hybrid chickens and other chickens can also suffer from the disease. The affected birds are dead more than 20-30 days of age, peaks at 28-35 days old, the period of death lasted 4-8 days, fatality decreases between 35-42 days old with duration of 8-15 days [8-9]. The mortality is about
$20 \%-75 \%$, up to $80 \%$ [10]. The HHS caused by FAV-4 strain (TJ1607) which is a non-encapsulated double-stranded DNA virus through epidemiological investigation, necropsy, morphological observation and genetic sequence analysis in Tianjin [11]. TJ1607 was $98.3 \%-97.3 \%$ in homology compared with FAV-4, among them, the homologous relationship with the isolated strain JSJ13 in China was 98.3\%, and the nucleic acid sequence was compared. The serious economic losses to the poultry industry in China were caused by HHS. Consequently, studies in pathogenicity and control are of great significance for protecting and promoting the development in poultry industry in China.

\subsection{Disease Model}

The Pathogenicity to one-day-old chicks with TJ1607 was explored by viral allantoic fluid and liver homogenate supernatant was infected into the 1-day-old chicks by dropping nose, eye and orally in the paper. The symptoms, mortality, gross lesions and histopathological changes were observed daily with post-inoculation, meanwhile the virus in tissue and hydropericardium was detected by PCR to be sure the same as TJ1607. The results showed that successful disease model was constructed for 1-day-old chicks by TJ1607, and the virus accumulation tissues were liver and heart. FAV-4 is acid-tolerant virus, can reach the small intestine through the stomach and is phagocytized by small intestinal epithelial cells, meanwhile virus can replicate in small intestine epithelial cells and enters the bloodstream via the small intestine central chylotrage to reaches the liver on the basis of Saifuddin et al. In addition, free virus in the blood can be taken and proliferate by hepatocytes to cause liver damage within 4-7 days after infection. Therefore, the liver is the main target organ for the virus to invade firstly, and play an important role in virus replication. The results of the study also demonstrate this point of view [12].

\subsection{Law of Incidence in Disease Model}

The clinical symptoms were depression, loss of appetite, lethargy and yellow loose stools in the early stage of infection with TJ1607. Subsequently, rapid death and high mortality were appeared in the experimental group. The macroscopic examination results show that hydropericardium and hepatitis with large amounts of basophilic intranuclear inclusions obviously in the dead chicks. The histopathological examination results show that inflammatory cell infiltration in the liver and widened interstitial edema of the myocardium in the dead chicks. The above reactions that the responses to antiviral damage in the body show that FAV-4 cause damage to the immune organs and immunosuppression in chickens [13]. FAV-4 has high tropism on chicken hepatocytes, and pathological changes in liver can reflect the strength of the virus in chickens [14]. The results of clinical manifestations and pathological changes after infection with TJ1607 in the absence of interference from maternal antibodies and other viruses in the study were similar with research reports by other scholars such as Abdul-Aziz TA and Mac Ferran [15-16]. 


\subsection{The Virus Mapping of Disease Model}

PCR results show the distribution of TJ1607 are both in the heart and liver which are consistent with the results of macro-morphological examination and histopathological examination with TJ1607 post-inoculation. The liver can be used as the primary choice for the clinical detection of FAV-4 due to the highest titer of virus location.

\section{Conclusion}

The HHS on a chicken farm in Tianjin for the firstly reported in July 2016. The result show that pathogen causing the disease was FAV-4 TJ1607 strain in Tianjin (genebank accession number: KY885021) which isolated and identified from dead \& sick birds on a chicken farm. HHS was replicated successfully through inoculated to one-day-old chicks with TJ1607 by simulate natural infection. The pathogenesis of the disease was determined, and the pathogenicity of FAV-4 Tianjin strain was studied in the paper. The theoretical basis for comprehensive prevention and treatment will be provided in poultry industry.

\section{References}

[1] Yin Zhen, Liu Jinghua. Animal virology [M]. Version 2. Beijing: Science Press, 1997.

[2] Calnek BW, Barns HJ, Beard CW. Diseases of poul-try [M]. 10th ed, Ames, IA, USA: Iowa State Univer.

[3] Wang Jing, Zhang Wensheng, Liu Rui, Li Fugui. Avian pericardial effusion-hepatitis syndrome in Tianjin and its virus isolation and identification [J]. Veterinary Medicine of China, 2017, 53(3):16-17.

[4] Xu Jinghui, Lou Shaosong, Tan Qiaoyan, Huang Jiachao. Methods for the establishment of animal models of hepatic injury and progress in molecular mechanisms. Seventh South China Regional Laboratory Animal Science and Technology Exchange Proceedings.
[5] He Haijian. 2001. Techniques and inspection points of carcass necropsy. Shandong poultry, 1(4), 16-17.

[6] Li Quan. 2011. Method of chicken body dissection. Pig industry observation (5), 29-30.

[7] Zhao J, Zhong Q, Zhao Y. Pathogenicity and complete genome characterization of fowl adenoviruses isolated from chickens associated with inclusion body hepatitis and hydropericardium syndrome in China [J]. PLos One, 2015, 10(7): e0133073.

[8] Yan Youxiang. 2016. I. Prevalence and control of flock adenovirus infection. Northern Herd (10), 21-21.

[9] Gai Shuwen. 2016. Diagnosis and treatment of broiler chicken Ankara disease-pericardial effusion hepatitis syndrome. Chinese Livestock and Poultry Breeding, 12(9), 154-154.

[10] Liu Dianzuo. 2015. Prevention and treatment of pericardial hydrocephalus-hepatitis syndrome. China Animal Health (10), 49-50.

[11] Mcferran J B, Smyth J A. Avian adenoviruses. [J]. Revue Scientifique Et Technique, 2000, 19(2):589-601.

[12] Saifuddin M and Wilks C R. Pathogenesis of an acute viral hepatitis: inclusion body hepatitis in the chicken. Archives of Virology, 1991, (1):33-43.

[13] Saifuddin M and Wilks C R. Reproduction of nclusion body hepatitis in conventionally raised chickens inoculated with a New Zealand isolate of avian adenovirus. New Zealand Veterinary Journal, 1990, (2):62.

[14] Huang Bing. Expression of avian influenza H5 hemagglutinin avian adenovirus recombinant and its characteristics [D]. Nanjing: Nanjing Agricultural University, 2005.

[15] Abdul-Aziz T A, Hasan S Y. Hydropericardium syndrome in broiler chickens: its contagious nature and pathology [J], Res Vet Sci, 1995, (59):219-221.

[16] MACFERRAN J B, CALN EK B W, BERAD H J, MCDOUGALD L R. Group I adenovirus infections [M]. Diseases of poultry. 10th ed. Ames: IA Press, 1997:608-620. 